\title{
High Expression of hsMAD2 in the Villi of Spontaneously Aborted Embryo with Chromosomal Abnormality
}

\author{
Zaochang Wang1 ${ }^{*}$, Junjie $\mathrm{Hu}^{2 *}$, Wang $\mathrm{He}^{2}$, Humin Gong ${ }^{3}$, Weihua $\mathrm{Xu}^{2}$, Zhichao $\mathrm{Ma}^{2}$, \\ Xinping Chen" ${ }^{2 \#}$ \\ ${ }^{1}$ Clinical Laboratory, Nanda Town Hospital of Danzhou City, Danzhou, China \\ ${ }^{2}$ Central Laboratory, Hainan General Hospital, Hainan Hospital Affiliated to The Hainan Medical College; Hainan Provincial Key \\ Laboratory of Cell and Molecular Genetic Translational Medicine, Haikou, China \\ ${ }^{3}$ Department of Obstetrics, Hainan General Hospital, Hainan Hospital Affiliated to The Hainan Medical College, \\ Haikou, China \\ Email: ${ }^{\circ}$ chenxinping52@126.com
}

How to cite this paper: Wang, Z.C., Hu, J.J., He, W., Gong, H.M., Xu, W.H., Ma, Z.C. and Chen, X.P. (2021) High Expression of hsMAD2 in the Villi of Spontaneously Aborted Embryo with Chromosomal Abnormality. Open Journal of Obstetrics and Gynecology, 11, 763-772.

https://doi.org/10.4236/ojog.2021.116071

Received: May 7, 2021

Accepted: June 15, 2021

Published: June 18, 2021

Copyright $\odot 2021$ by author(s) and Scientific Research Publishing Inc. This work is licensed under the Creative Commons Attribution International License (CC BY 4.0).

http://creativecommons.org/licenses/by/4.0/

(c) (i) Open Access

\begin{abstract}
Objective: To investigate the changes of hsMAD2 protein and gene expression levels during chromosome segregation of human embryos. Method: The embryos of spontaneous abortion were collected in our hospital from 2009 to 2013, the chromosomal numbers of the embryonic villi were subsequently detected by fluorescence in situ hybridization (FISH). The patients were then divided into the normal and abnormal groups based on the chromosome number. The hsMAD2 protein and gene expression levels in the villi tissues of the two embryo groups were detected by western blotting and qRT-PCR, respectively. The hsMAD2 protein and gene levels in the embryonic villus tissue of the patient were detected. Results: From 2009 to 2013, we collected 50 embryos from spontaneous abortion patients. The chromosome abnormality and no abnormality were 36 cases (abnormal number of 28 cases (56.0\%) and chimerism in 8 cases $(16.0 \%)$ ) and 14 cases (28.0\%), respectively. The expression of hsmad2 protein and its gene in the villi of spontaneously aborted embryo with chromosomal abnormality in the abnormal group was significantly higher than that in those without chromosomal abnormalities $(0.88 \pm 0.20$ vs $0.61 \pm 0.19, \mathrm{P}<0.05),(23.46 \pm 0.07$ vs $18.35 \pm 0.10, \mathrm{P}<0.05)$. Conclusion: Abnormal number of chromosomes is closely related to spontaneous abortion Linked, hsMAD2 factor has a card effect on the cell cycle, can block the mitotic process of cells, and play an important role in maintaining the normal separation and stability of chromosomes.
\end{abstract}

*These authors contribute equally to this work.

\#Corresponding author. 


\section{Keywords}

Spontaneous Abortion, Embryonic Villi, Chromosomal Abnormality, hsMAD2

\section{Introduction}

Spontaneous abortions account for $15 \%$ - 18\% of clinically recognized pregnancies. Its occurrence is mainly related to known factors such as heredity, infection, immunity, and environment, as well as other unknown factors [1] [2]. Human embryonic development is a gradual process. After the fertilization of the ovum, the gene of the fertilized ovum is reprogrammed. After the division, the archiblast is distributed into the daughter cells, which leads to the formation of early embryos. The early embryo lacks transcriptional activity; the embryonic gene is gradually formed and activated with the development of the embryo. Chromosomal abnormalities may occur during any of these processes. Therefore, the integrity of reprogramming, continuous cleavage, and formation and activation of the embryonic genome must be maintained for normal development of the human embryo [3] [4] [5]. Mitosis is a continuous process of nuclear restitution, and a basic form of realizing histogenesis and individual development. The spindle assembly checkpoint (SAC) prevents chromosome segregation. The cell cannot enter mitosis anaphase, and continue into normal cell cycle activities, until the sister chromatid is properly connected to the mitotic spindle [6] [7]. There is no correlation between hsmad 2 and chromosomal abnormalities in spontaneous abortion embryos at home and abroad. Therefore, we collected the villi tissue in spontaneous abortion patients in our hospital from March 2009 to October 2013. Compare the expression of hsmad 2 protein and gene between chromosomal abnormalities and normal fetal chorionic villi, in order to explore the effect of SAC on spontaneous abortion resulting from chromosomal abnormalities.

\section{Material and Methods}

\subsection{General Data}

The 50 placental villi tissues treated for spontaneous abortion were collected for this study at the Obstetrics and Gynecology department of the Hainan Province People's Hospital between March 2009 and October 2013. These samples were collected from pregnant women with spontaneous abortion who excluded history of infection and exposure to harmful poisons, and the placentas of all patients were confirmed to have stopped developing by the b-scan ultrasonic method. This project was approved and implemented by the Ethics Committee of Hainan Provincial General Hospital, and the number is Med-Eth-Re [2021] 131. 


\subsection{Detection Method}

\subsubsection{Detection of Embryonic Villi Chromosomes by FISH}

The embryonic villi were rinsed with $0.9 \% \mathrm{NaCl}$ solution, pre-fixed, and subsequently fixed. The treated specimens were prepared into a stationary liquid, and the cell suspension stained on a glass slide, which was placed on an aging slide of $56^{\circ} \mathrm{C}$ for over 2 hours. The samples were rinsed, digested, and dewatered, and then subjected to in situ hybridization in a hybridization oven at $42^{\circ} \mathrm{C}$ for 17 hours; this process was repeated twice. The slides were warmed for 30 minutes, cleaned, and subsequently stained. The slides were then observed under a fluorescent microscope.

\subsubsection{Detection of hsmad2 Protein by Western Blotting}

One milliliter of lysis buffer was added to $200 \mathrm{mg}$ embryonic villi tissues. The samples were ground to a smooth past, and incubated for 30 minutes. The supernatant was removed, and the samples centrifuged at $4^{\circ} \mathrm{C}$ for 5 minutes at a speed of 13,000 rpm. The supernatant was discarded again. Supernatant buffer $(25 \mu \mathrm{L})$ was then added to $100 \mu \mathrm{L}$ specimen that was to be tested. The samples were denatured in a $100^{\circ} \mathrm{C}$ water bath, and subjected to sodium dodecyl sulfate polyacrylamide gel electrophoresis (SDS-PAGE); the separated proteins were transferred onto a polyvinylidene difluoride (PVDF) membrane and sealed with $5 \%$ nonfat dried milk for 2 hours. The membrane was then incubated with anti-hsMAD2 and $\beta$-actin $(1: 1000)$ antibodies overnight at $4^{\circ} \mathrm{C}$. Subsequently, the membrane was incubated with the corresponding secondary antibodies (1:5000) for 60 minutes at room temperature. The PVDF membrane was placed on an image scanner, and covered with a previously prepared developing-coloring solution (in the dark). The image-lab software was run in order to analyze and process, 1 minute after the reaction.

\subsubsection{Detection of hsMAD2 mRNA by qRT-PCR}

According to the designed primer sequences (submitted to NCBI GenBank), the length of the amplified fragment was about $100-300 \mathrm{bp}$. The specific primer for hsMAD2 was synthesized by Sangon Biotech Co., Ltd. (Shanghai, China). The sequences were as follows, hsMAD2-F: 5'-AGGGTTTTGAAGCCGTCTAT-3', hsMAD2-R: 5'-CGTCTGCCTTCGGTATTCT-3', GADPH-F:

5'-CCCACTCCTCCAC-CTTTG-3', GADPH-R:

5'-CCACCACCCTGTTGCTG-3'. The samples were soaked in $0.1 \%$ diethy pyrocarbonate (DEPC) solution at room temperature overnight or processed at $37^{\circ} \mathrm{C}$ in a $1.5 \mathrm{~mL}$ Eppendorf tube. Tips of varying capacities $(10 \mu \mathrm{L}, 200 \mu \mathrm{L}$, and $1 \mathrm{~mL}$ ) were sterilized using a high temperature stream and autoclaved at $80^{\circ} \mathrm{C}$ standby. Embryonic villi tissue $(300 \mathrm{mg}$ ) was added to $4.5 \mathrm{~mL}$ TRIzol extracting solution, and the mixture centrifuged at $12000 \mathrm{rpm}$ after rapid grinding on ice. The supernatant was collected in another centrifuge tube, treated with $1 \mathrm{~mL}$ chloroform, and oscillated for 15 seconds. This was placed on ice for 5 minutes, and subsequently centrifuged again at $4^{\circ} \mathrm{C}$ and $12,000 \mathrm{rpm}$ for 15 minutes. Again, 
the supernatant was collected in another centrifuge tube; this was treated with 3 $\mathrm{mL}$ isopropanol, and mixed uniformly. This mixture was placed on ice for 15 minutes and centrifuged again at $12,000 \mathrm{rpm}$ and $4^{\circ} \mathrm{C}$ for 15 minutes. The supernatant was discarded and the pellet treated with $1 \mathrm{~mL} 75 \%$ ethanol. This was centrifuged again at $7500 \mathrm{rpm}$ and $4^{\circ} \mathrm{C}$ for 5 minutes. The ethanol solution was removed, and the pellet placed on ice for 10 minutes. The total RNA was dissolved using DEPC $-\mathrm{H}_{2} \mathrm{O}$, and subjected to reverse transcription. The samples were reverse transcribed using the following program: $95^{\circ} \mathrm{C}$ ( 6 minutes) $\rightarrow 55^{\circ} \mathrm{C}$ (90 seconds) $\rightarrow 72^{\circ} \mathrm{C}$ (90 seconds) $\rightarrow 72^{\circ} \mathrm{C}$ ( 10 minutes $) \rightarrow 5^{\circ} \mathrm{C}$ ( 1 hour). The real-time polymerase chain reaction (PCR) was performed in accordance with the manufacturer instructions. GAPDH and its copy number were taken as the internal reference and correction base, respectively; the cycle threshold (CT) value was obtained using the ABI7500 software (Applied Biosystems, Foster City, CA, USA). The $\Delta \mathrm{C}_{\mathrm{T}}$ value (corresponding threshold of the specimen) was obtained by subtracting the obtained $\mathrm{C}_{\mathrm{T}}$ value from the $\mathrm{C}_{\mathrm{T}}$ value of GAPDH of the same specimen. The $\Delta \mathrm{C}_{\mathrm{T}}$ value of the sham operation group was taken as the correction base, and the $\mathrm{C}_{\mathrm{T}}$ gene concentration level was obtained by software calculation using the obtained ${ }^{2-\Delta \Delta} \mathrm{C}_{\mathrm{T}}$ value.

The kits used and the operating methods of FISH detection, $\mathrm{Wb}$ detection and qRT-PCR detection are all provided by Lulong Biotechnology Development Co., Ltd. (Xiamen, China); And all processes are carried out in strict accordance with the manufacturer's instructions.

\subsection{Statistical Analysis}

The obtained data was statistically analyzed using SPSS software (v.16.0, IBM, Armonk, NY, USA). All data was presented as mean \pm standard deviation (S.D.). Significant differences were determined by analysis of variance (ANOVA). The difference was considered to be statistically significant when P-values were $<0.05$.

\section{Results}

\subsection{Comparison of General Conditions}

From 2009 to 2013, we collected 50 embryos from spontaneous abortion patients. The chromosome abnormality and no abnormality were 36 cases (abnormal number of 28 cases (56.0\%) and chimerism in 8 cases (16.0\%)) and 14 cases $(28.0 \%)$, respectively. Two groups of pregnant women were comparable as no statistical difference $(\mathrm{P}>0.05)$ was observed (Table 1$)$.

\subsection{FISH Detection}

Twenty-eight embryos with chromosome abnormalities were detected in embryos of 50 patients with spontaneous abortion (56\%; 28/50). Eight chimeras (16\%) were detected among these. The details of abnormal chromosome types are provided in Table 2 and Figure 1. 
Table 1. Comparison of general situation between two groups.

\begin{tabular}{cccc}
\hline group & gestational age (years) & Pregnancy (second) & Gestational week (week) \\
\hline Abnormal group & $28.96 \pm 4.59$ & $2.41 \pm 1.52$ & $10.77 \pm 2.31$ \\
Normal group & $29.04 \pm 4.61$ & $2.50 \pm 1.28$ & $10.58 \pm 2.42$ \\
P value & 0.32 & 0.18 & 0.25 \\
T value & 3.41 & 3.52 & 4.44 \\
\hline
\end{tabular}

Table 2. Specific distribution of chromosomal abnormal nuclei (example).

\begin{tabular}{ccc}
\hline type & Abnormal chromosome number (example) & detection rate (\%) \\
\hline triploid & 8 & 16 \\
$45, \mathrm{X}$ & 5 & 10 \\
16 -trisomic & 4 & 8 \\
18 -trisomic & 3 & 6 \\
22 -trisomic & 3 & 6 \\
13 -trisomic & 2 & 4 \\
21 -deficiency & 1 & 2 \\
21 -trisomic & 1 & 2 \\
$47, \mathrm{XXY}$ & 1 & 2 \\
\hline
\end{tabular}
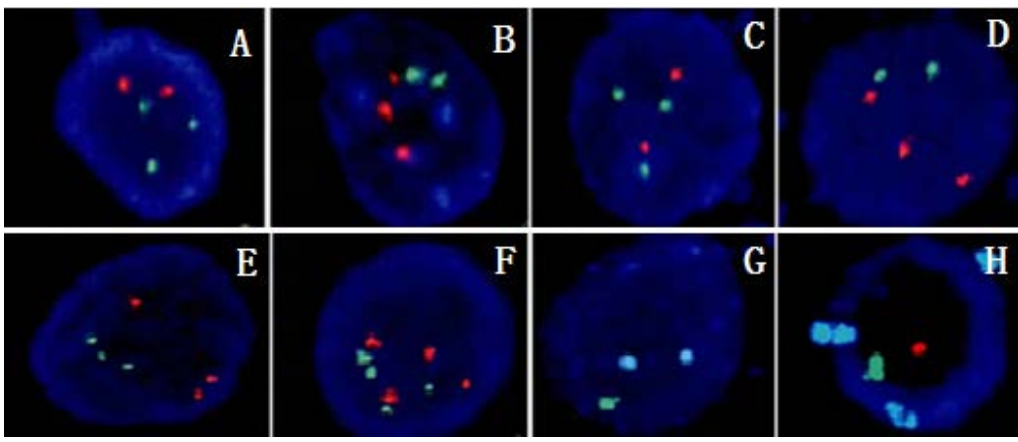

Figure 1. Fish detection of chromosomal abnormalities in different groups of embryos. Note: A, B Green fluorescent signal is chromosome 13., Red fluorescence signal is chromosome 21. C, D, E, F Green fluorescence signal is chromosome 16, red signal is chromosome 22. G, H Green fluorescence signal is $\mathrm{x}$ chromosome, Red fluorescence signal is $\mathrm{y}$ chromosome, Sky blue signal is chromosome 18. Description: 13-disome, 21-trisomic, 16-disome, 22-disome, triploid, tetraploid, X-monomer, 18-trisomic.

\subsection{Comparison of Relative Expression Levels of hsMAD2 Protein in Spontaneously Aborted Embryonic Villi}

The relative expression levels of the hsMAD2 protein in abnormal embryonic villi tissues was higher than those seen in the control group (Table 3 and Figure 2).

\subsection{Comparison of Relative Expression Levels of hsMAD2 Gene in Spontaneously Aborted Embryonic Villi}

The relative expression levels of $h s M A D 2$ gene was much higher in the abnormal embryo villi tissues than in the control group, as shown in Table 4 and Figure 3 . 


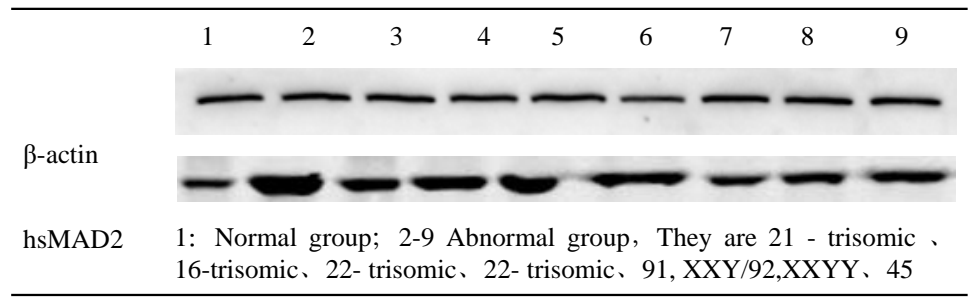

Figure 2. Comparison of the relative expression of hsMAD2 protein in two groups.

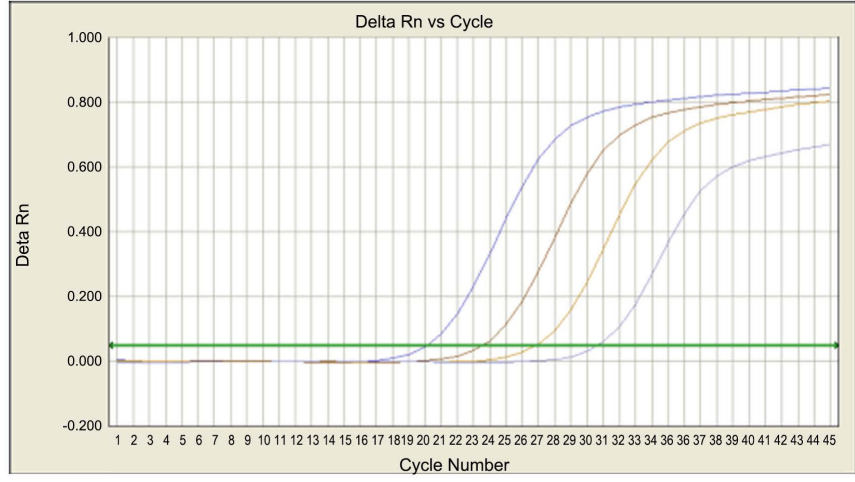

Hsmad2 standard curve amplification

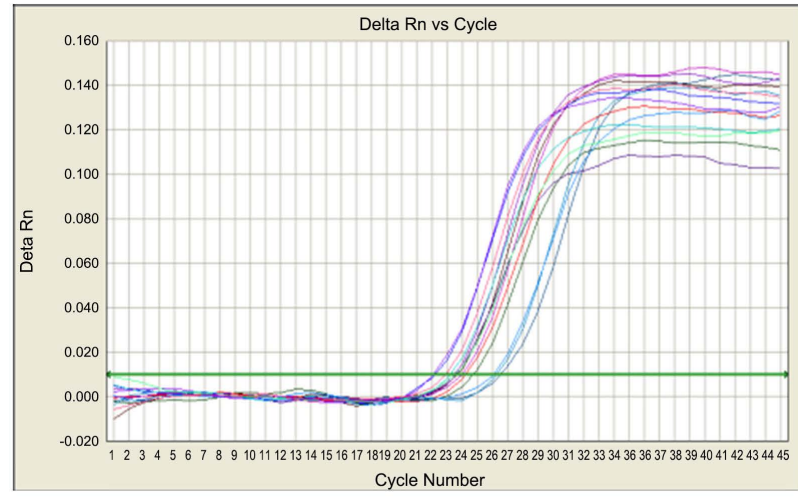

Hsmad2 amplification curve

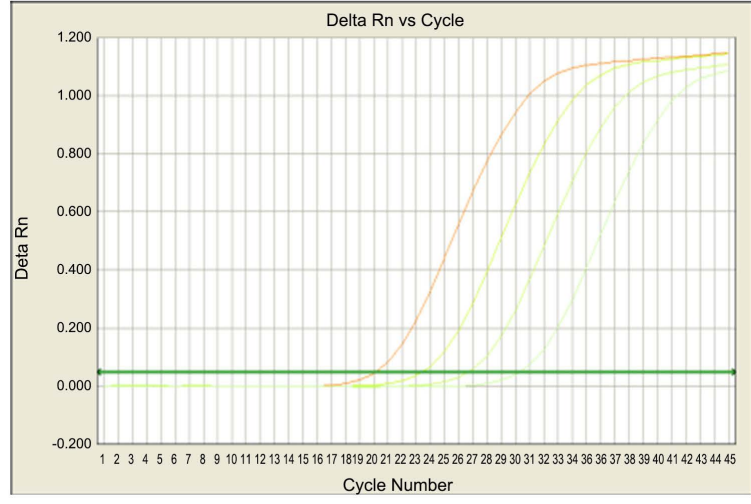

GADPH standard curve amplification

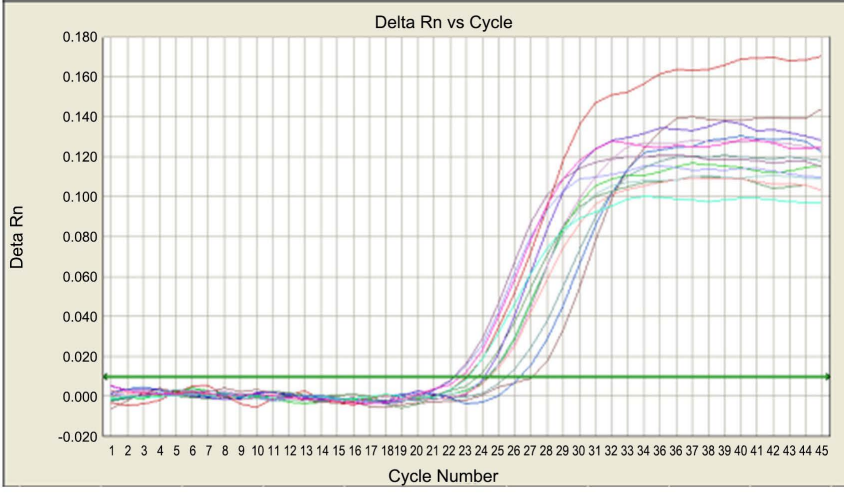

GADPH amplification curve

Figure 3. Comparison of relative expression of hsmad2 gene between two groups. Note: the amplification curve of internal reference is smooth and no non-specific amplification, indicating the primer synthesis and experimental system design standard.

Table 3. Comparison of relative expression of hsmad2 protein between two groups.

\begin{tabular}{ccc}
\hline type & Number of examples & Protein relative expression \\
\hline Abnormal group & 28 & $0.88 \pm 0.20$ \\
Normal group & 53 & $0.61 \pm 0.19$ \\
P value & & 0.002 \\
T value & 4.79 \\
\hline
\end{tabular}

Table 4. Comparison of hsmad2 gene changes between two groups.

\begin{tabular}{cccc}
\hline type & CT (hsMAD2) & CT (GAPDH) & $2-\Delta \Delta$ CT \\
\hline Abnormal group & $23.46 \pm 0.07$ & $21.90 \pm 1.63$ & $3.54 \pm 1.01$ \\
\hline
\end{tabular}


Continued

\begin{tabular}{ccc}
\hline Normal group & $18.35 \pm 0.10$ & $22.89 \pm 1.55$ \\
P value & 0.019 \\
T value & 3.59 \\
\hline
\end{tabular}

\section{Discussion}

Spontaneous abortion is a term used to describe an abortion caused by nonhuman operation. Clinical research confirms its correlation to multiple factors, including heredity and immunity. In 1983, Simoni first proposed a connection between spontaneous abortion and chromosome abnormalities; this led to a greater research focus on this subject. The latest data indicates that the rate of spontaneous abortion in pregnant women at gestational weeks $<6$ is about $50 \%$; among these $70 \%$ are believed to be a direct result of chromosomal abnormalities. On the other hand, the rates of spontaneous abortion in pregnant women at 6 - 10 and $>10$ gestational weeks were $15 \%$ and only $3 \%$ (50\% and $5 \%$ related to chromosomal abnormality), respectively. This indicated that spontaneous abortion caused by chromosomal abnormalities takes place easily during the early stages of pregnancy [8] [9]. In this study, chromosomal abnormalities were detected in 28 out of 50 embryos (56\%) obtained from patients who underwent spontaneous abortion, indicating that more than half of the embryos with chromosomal disease will be naturally eliminated and cannot survive up to birth. This morbidity rate is consistent with the results obtained in previous studies [2] [10]. After comparing pregnant women with chromosomal abnormalities and normal pregnant women, this study excluded miscarriage caused by basic factors such as gestational age, gestation time, and gestational age [11].

There are two types of chromosomal abnormalities: numerical abnormality and structural abnormality. The clinical incidence rate of numerical abnormality, especially aneuploidy, is generally higher. The occurrence of aneuploid is mainly a result of the non-disjunction of gametes during the initial stages of fertilized ovum formation, which causes an increase or decrease in the chromosome number. Aneuploid chromosome abnormalities greatly influence the embryo; the resulting fatality rate is also very high. Therefore, it is one of the most common clinical types. The incidence rate of aneuploidy was found to be $68 \%$ in this study, which was much higher than that of polyploidy (20\%) and mosaicism (12\%). This data is consistent with the results obtained in previous clinical studies.

The chromosome goes through periodic changes (condensation and stretching) during the cell cycle. Therefore, chromosomal abnormalities may occur at any link of the cell cycle. The cell cycle checkpoint is a regulatory mechanism of the cell cycle. A normal cell cycle has 3 checkpoints: 1) the G1 $\rightarrow$ S stage, where one must check if the cell has developed enough for chromosome replication, or if the cell has left the cell cycle and entered the resting stage; 2) the S $\rightarrow$ G2 stage, where one can check to see if all chromosomes have been replicated; 3) a check- 
point during mitosis, wherein one must confirm that all spindles are connected to the chromosomes before continuing on to mitosis. In addition, one can analyze whether the SAC makes a full impact during the spindle and chromosome docking and segregation [12] [13] [14]. Therefore, the cell cycle can be effectively monitored by detecting the SAC. hsMAD2 is the checkpoint gene for monitoring the spindle activity performance. MAD2 is collected in the kinetochore of the chromosome after activation. Here, its conformation changes, leading to the formation of C-MAD2. C-MAD2 interacts with Cdc20, BUBR1, and BUB3, and forms a mitotic checkpoint complex, which provides a correct point in time for the complete docking of chromosomes during cell division. Previous studies have also reported alternative methods for the regulation of the docking and segregation of chromosomes, using methods other than the SAC. hsMAD2 can act on mitotic kinesin-like protein 2 (MKlp2) during mitosis, which can induce cytokinesis. The chromosomes convey complexes driven by MKlp2. The ability of MKlp2 to reload to the complexes from the centromere is restrained by hsMAD2, which reduces the early ineffectiveness of the SAC, as well as any increase in cytokinesis errors [15] [16]. Therefore, we attempted to detect the hsMAD2 gene and protein expression, as an indirect means of observing the effect of the SAC, assuming hsMAD2 to be a link between SAC and chromosome abnormality. The spindle behavior was indirectly monitored by detecting the variations in the level of hsMAD2.

The hsMAD2 protein and gene expression levels were detected by western blotting and qRT-PCR; the expression of the hsMAD2 protein and gene was higher in the embryonic villi tissues of patients with spontaneous abortion resulting from chromosomal abnormalities than those in patients without any such abnormalities. This led to the speculation that docking and segregation errors during the mitosis of fertilized ovum could result in chromosomal numerical abnormalities in the embryonic tissue. The cells with chromosomal numerical abnormalities continue the cell division. A greater amount of hsMAD2 is provided by the organism to guarantee the correct division of ligand chromosomes into the daughter cells. This ensures that sufficient time is provided for the SAC, and that the MKlp2 is sufficiently restrained to reload to the complex from the centromere. This facilitates the smooth transition of the cell into the mitotic anaphase. Therefore, SAC maintains the integrity of genome by increasing the concentration of hsMAD2.

In conclusion, our data suggests that chromosomal numerical abnormalities play a major role in spontaneous abortion. hsMAD2 expression is higher when spontaneous abortion occurs as a result of chromosomal abnormalities. Its blocking function in mitosis plays an important role in maintaining the integrity of the chromosome. However, our research still has certain shortcomings: the number of samples is not large enough, and we should try to collect the subjects of spontaneous abortion with a large gestational week. In addition, whether there is a difference in the expression of the hsMAD2 gene in normal people at different gestational weeks, and whether it is normal in spontaneous abortion, whether 
there is a difference in the expression of hsMAD2 gene between the group and the normal unnatural abortion population.

\section{Acknowledgements}

This work was supported by a grant provided by The Youth Science and Technology Talents Academic Innovation Program Project of Hainan Association for Science and Technology (QCXM201921).

\section{Conflicts of Interest}

The authors declare no conflict of interest.

\section{References}

[1] Zhang, Z.-P., Xing, W.-W., Zhang, B.-B., Liang, L.-X., Zhu, L. and Wu, X.-Q. (2016) Expression of Centromere Protein-C (CENP-C) in Villus Tissue of the First-Trimester Spontaneous Abortion and Its Correlation with Chromosome Segregation. Journal of Reproduction \& Contraception, 27, 18-24.

[2] Erdal, T., Nilgün, T., Osman, D., Dilara, S. and Nesrin, Ç. (2016) Chromosomal Analyses of 1510 Couples Who Have Experienced Recurrent Spontaneous Abortions. Reproductive BioMedicine Online, 32, 414-419. https://doi.org/10.1016/j.rbmo.2016.01.006

[3] Ruth, D.P. and Liu, J. (2017) On the Robustness of SAC Silencing in Closed Mitosis. Biophysical Journal, 3, 291A. https://doi.org/10.1016/j.bpj.2016.11.1578

[4] Lian, A.T.Y. and Chircop, M. (2016) Mitosis in Animal Cells. Encyclopedia of Cell Biology, 3, 479-493. https://doi.org/10.1016/B978-0-12-394447-4.30064-5

[5] Del Carmen Nogales, M., Bronet, F., Basile, N., María Martínez, E., Liñán, A., Rodrigo, L., et al. (2017) Type of Chromosome Abnormality Affects Embryo Morphology Dynamics. Fertility and Sterility, 107, 229-235.E2.

[6] Deng, G.S., Zhang, J.G. and He, J. (2013) Chromosome Karyotypes Analysis of 171 Couples with Spontaneous Abortion in Yulin Region. Chinese Journal of Birth Health \& Heredity, 125, 61-62.

[7] Meng, X.N. and Xu, F. (2013) Gene Chip Detection Preliminary Analysis of Peripheral Blood of Patients with Recurrent Abortion. Prenatal Diagnosis, 6, 48-49.

[8] Lee, S.H., McCormick, F. and Saya, H. (2010) Mad2 Inhibits the Mitotic Kinesin MKlp2. Journal of Cell Biology, 191, 1069-1077.

https://doi.org/10.1083/jcb.201003095

[9] Wei, R.M. (2013) Study on Relationship between Recurrent Spontaneous Abortion and Feminine Genital Tract Mycoplasma Infection. Journal of Baotou Medicine, 8 , 87-88.

[10] Nonaka, T., Ooki, I., Enomoto, T. and Takakuwa, K. (2015) Complex Chromosomal Rearrangements in Couples Affected by Recurrent Spontaneous Abortion. International Journal of Gynecology \& Obstetrics, 128, 36-39.

https://doi.org/10.1016/j.ijgo.2014.07.018

[11] Hassold, T. and Hunt, P. (2009) Maternal Age and Chromosomally Abnormal Pregnancies: What We Know and What We Wish We Knew. Current Opinion in Pediatrics, 21, 703-708. https://doi.org/10.1097/MOP.0b013e328332c6ab

[12] Da, Y.J. (2013) Change and Significance of CA125 Level in Early Pregnancy Serum 
of Patients with Spontaneous Abortion. Journal of Ningxia Medical University, 8, 465-467.

[13] Mu, Y.Q., Liu, R.H., Zhu, Z.Y., Wang, J., Li, S.M., Wang, Y.H. and Zhao, F.X. (2013) Research on Role of Heparanase in Process of Human Spontaneous Abortion. Family Planning, 9, 13-15.

[14] Kawashima, S.A., Yamagishi, Y., Honda, T., Ishiguro, K. and Yoshinori, W. (2010) Phosphorylation of H2A by Bub1 Prevents Chromosomal Instability through Localizing Shugoshin. Science, 327, 172-177. https://doi.org/10.1126/science.1180189

[15] Xing, J.F., Jia, L.T., Wu, Y.L., Zhang, Z., Cui, S.H., Cheng, G.M., et al. (2012) Expressions of MAD2 and BUB1 Genes in Spontaneously Aborted Embryo with Chromosome Numerical Abnormality. Reproduction and Contraception, 32, 349-354.

[16] Fragouli, E., Lenzi, M., Ross, R., Katz-Jaffe, M., Schoolcraft, W.B. and Wells, D. (2008) Comprehensive Molecular Cytogenetic Analysis of the Human Blastocyst stage. $\mathrm{Hu}$ man Reproduction, 23, 2596-608. https://doi.org/10.1093/humrep/den287 